\title{
Dyadic Green Function for an Electromagnetic Medium Inspired by General Relativity
}

\author{
Akhlesh Lakhtakia ${ }^{1 *}$, Tom G. Mackay ${ }^{\dagger}$ \\ ${ }^{1}$ CATMAS, Department of Engineering Science and Mechanics, Pennsylvania State University, \\ University Park, PA 16802-6812, USA \\ ${ }^{2}$ School of Mathematics, University of Edinburgh, Edinburgh EH9 3JZ, United Kingdom
}

(Received 00 November 2005)

The dyadic Green function for a homogeneous electromagnetic medium inspired by the spatiotemporally nonhomogeneous constitutive equations of gravitationally affected vacuum is derived.

PACS: 41.20.-q, 41.20.Jb, 78.20.-e

\footnotetext{
*E-mail: akhlesh@psu.edu

${ }^{\dagger}$ Corresponding author. E-mail: T.Mackay@ed.ac.uk
} 
Vacuum or matter-free space is the most widely studied electromagnetic medium, not only because it underlies the development of continuum electromagnetic properties from microscopic principles $[1$, $2]$, but also because of the significance of electromagnetic communication devices in modern society [3]. The electromagnetic constitutive equations of vacuum are commonly stated in textbooks as

$$
\begin{aligned}
& \mathbf{D}(\mathbf{r}, t)=\epsilon_{0} \mathbf{E}(\mathbf{r}, t), \\
& \mathbf{B}(\mathbf{r}, t)=\mu_{0} \mathbf{H}(\mathbf{r}, t),
\end{aligned}
$$

where $\epsilon_{0}=8.854 \times 10^{-12} \mathrm{~F} \mathrm{~m}^{-1}$ and $\mu_{0}=4 \pi \times$ $10^{-7} \mathrm{H} \mathrm{m}^{-1}$ in SI units, whereas $\mathbf{r}$ and $t$ indicate position and time.

These equations presuppose either the absence of a gravitational field or that the observer is local. When a gravitational field is present, spacetime appears curved — which is well-known [4]. One can still use the textbook versions of the Maxwell postulates for gravitationally affected vacuum, but the constitutive relations are now $[5,6]$

$$
\begin{aligned}
\mathbf{D}(\mathbf{r}, t) & =\epsilon_{0} \underline{\underline{\gamma}}(\mathbf{r}, t) \cdot \mathbf{E}(\mathbf{r}, t) \\
& -c_{0}^{-1} \boldsymbol{\Gamma}(\mathbf{r}, t) \times \mathbf{H}(\mathbf{r}, t), \\
\mathbf{B}(\mathbf{r}, t) & =\mu_{0} \underline{\underline{\gamma}}(\mathbf{r}, t) \cdot \mathbf{H}(\mathbf{r}, t) \\
& +c_{0}^{-1} \boldsymbol{\Gamma}(\mathbf{r}, t) \times \mathbf{E}(\mathbf{r}, t),
\end{aligned}
$$

in lieu of (1) and (2). Here $\underline{\underline{\gamma}}(\mathbf{r}, t)$ is a real symmetric dyadic and $\boldsymbol{\Gamma}(\mathbf{r}, t)$ is a vector with real-valued components, both related to the metric of spacetime; whereas $c_{0}=1 / \sqrt{\epsilon_{0} \mu_{0}}$.

Just as isotropic dielectric--magnetic mediums provide material counterparts of (1) and (2) in the frequency domain $[1,7]$, the vast variety of complex materials $[8,9]$ — natural as well as artificial - suggests that it is quite possible that (3) and (4) also have material counterparts. This thought inspired the present communication, wherein we present the derivation of the dyadic Green function for frequency-domain electromagnetic fields in a homogeneous medium inspired by (3) and (4).

With the assumption that all fields have an $\exp (-i \omega t)$ time dependence, with $\omega$ as the angular frequency, the constitutive relations of the chosen medium are

$$
\begin{array}{r}
\mathbf{D}(\mathbf{r}, \omega)=\epsilon_{0} \underline{\underline{\gamma}}(\omega) \cdot \mathbf{E}(\mathbf{r}, \omega) \\
-c_{0}^{-1} \boldsymbol{\Gamma}(\omega) \times \mathbf{H}(\mathbf{r}, \omega) \\
\mathbf{B}(\mathbf{r}, \omega)=\mu_{0} \underline{\underline{\gamma}}(\omega) \cdot \mathbf{H}(\mathbf{r}, \omega) \\
+c_{0}^{-1} \boldsymbol{\Gamma}(\omega) \times \mathbf{E}(\mathbf{r}, \omega) .
\end{array}
$$

The coordinate system has been chosen such that $\underline{\gamma}(\omega)$ is diagonal, and from now onwards the depen$\overline{\bar{d}}$ ence on $\omega$ is implicit.

The frequency-domain Maxwell curl postulates in the chosen medium may be set down as

$$
\begin{aligned}
\nabla \times \mathbf{E}(\mathbf{r})= & i \omega\left[\mu_{0} \underline{\underline{\gamma}} \cdot \mathbf{H}(\mathbf{r})+c_{0}^{-1} \boldsymbol{\Gamma} \times \mathbf{E}(\mathbf{r})\right] \\
\nabla \times \mathbf{H}(\mathbf{r})= & -i \omega\left[\epsilon_{0} \underline{\underline{\gamma}} \cdot \mathbf{E}(\mathbf{r})-c_{0}^{-1} \boldsymbol{\Gamma} \times \mathbf{H}(\mathbf{r})\right] \\
& +\mathbf{J}(\mathbf{r}),
\end{aligned}
$$

where $\mathbf{J}(\mathbf{r})$ is the source electric current density. Our objective is to find the dyadic Green functions $\underline{\underline{G}}_{e}(\mathbf{r}, \mathbf{s})$ and $\underline{\underline{G}}_{m}(\mathbf{r}, \mathbf{s})$ such that

$$
\begin{aligned}
& \mathbf{E}(\mathbf{r})=i \omega \mu_{0} \iiint \underline{\underline{G}}_{e}(\mathbf{r}, \mathbf{s}) \cdot \mathbf{J}(\mathbf{s}) d^{3} \mathbf{s},(9) \\
& \mathbf{H}(\mathbf{r})=\iiint \underline{\underline{G}}_{m}(\mathbf{r}, \mathbf{s}) \cdot \mathbf{J}(\mathbf{s}) d^{3} \mathbf{s}
\end{aligned}
$$

with the integrations being carried out over the region where the source electric current density is nonzero.

To begin with, the substitution of (9) in (7) and comparison of the resulting expression with (10) yields

$\underline{\underline{G}}_{m}(\mathbf{r}, \mathbf{s})=\underline{\underline{\gamma}}^{-1} \cdot\left(\nabla \times \underline{\underline{I}}-i k_{0} \mathbf{\Gamma} \times \underline{\underline{I}}\right) \cdot \underline{\underline{G}}_{e}(\mathbf{r}, \mathbf{s})$,

where $k_{0}=\omega \sqrt{\epsilon_{0} \mu_{0}}$ and $\underline{\underline{I}}$ is the identity dyadic. Thus, an expression for only $\underline{\underline{G}}_{e}(\mathbf{r}, \mathbf{s})$ has to be found.

For that purpose, following Lakhtakia \& Welglhofer [10], we start by defining new fields and source current density as

$$
\begin{aligned}
& \mathbf{e}(\mathbf{r})=\mathbf{E}(\mathbf{r}) \exp \left(-i k_{0} \boldsymbol{\Gamma} \cdot \mathbf{r}\right) \\
& \mathbf{h}(\mathbf{r})=\mathbf{H}(\mathbf{r}) \exp \left(-i k_{0} \boldsymbol{\Gamma} \cdot \mathbf{r}\right) \\
& \mathbf{j}(\mathbf{r})=\mathbf{J}(\mathbf{r}) \exp \left(-i k_{0} \boldsymbol{\Gamma} \cdot \mathbf{r}\right)
\end{aligned}
$$

Hence, (7) and (8) respectively transform to

$$
\begin{aligned}
& \nabla \times \mathbf{e}(\mathbf{r})=i \omega \mu_{0} \underline{\underline{\gamma}} \cdot \mathbf{h}(\mathbf{r}) \\
& \nabla \times \mathbf{h}(\mathbf{r})=-i \omega \epsilon_{0} \underline{\underline{\gamma}} \cdot \mathbf{e}(\mathbf{r})+\mathbf{j}(\mathbf{r})
\end{aligned}
$$

Next, we make use of an affine transformation associated with the scaling of space as per [11]

$$
\tilde{\mathbf{r}}=\underline{\underline{\gamma}}^{1 / 2} \cdot \mathbf{r}
$$


where $\underline{\underline{\gamma}}^{1 / 2} \cdot \underline{\underline{\gamma}}^{1 / 2}=\underline{\gamma}$ and we recall that $\underline{\underline{\gamma}}$ is a dyadic $\overline{\overline{ }}$ with real-valued elements. Let us define another set of fields and source current density as

$$
\begin{aligned}
& \tilde{\mathbf{e}}(\mathbf{r})=\underline{\underline{\gamma}}^{1 / 2} \cdot \mathbf{e}\left(\underline{\underline{\gamma}}^{1 / 2} \cdot \mathbf{r}\right) \\
& \tilde{\mathbf{h}}(\mathbf{r})=\underline{\underline{\gamma}}^{1 / 2} \cdot \mathbf{h}\left(\underline{\underline{\gamma}^{1 / 2}} \cdot \mathbf{r}\right) \\
& \tilde{\mathbf{j}}(\mathbf{r})=\left(\underline{\operatorname{adj}} \underline{\underline{\gamma}}^{1 / 2}\right) \cdot \mathbf{j}\left(\underline{\underline{\gamma}}^{1 / 2} \cdot \mathbf{r}\right),
\end{aligned}
$$

where 'adj' stands for the adjoint. Then, (15) and (16) transform to

$$
\begin{aligned}
& \nabla \times \tilde{\mathbf{e}}(\mathbf{r})=i \omega \mu_{0} g \tilde{\mathbf{h}}(\mathbf{r}) \\
& \nabla \times \tilde{\mathbf{h}}(\mathbf{r})=-i \omega \epsilon_{0} g \tilde{\mathbf{e}}(\mathbf{r})+\tilde{\mathbf{j}}(\mathbf{r})
\end{aligned}
$$

where

$$
g=\sqrt{\underline{\underline{\mid \gamma} \mid}}
$$

and $|\underline{\underline{\gamma}}|$ denotes the determinant of $\underline{\underline{\gamma}}$.

From the foregoing equations, we obtain

$$
\begin{aligned}
& {\left[(\nabla \times \underline{\underline{I}}) \cdot(\nabla \times \underline{\underline{I}})-k_{0}^{2} g^{2} \underline{\underline{I}}\right] \cdot \nabla \times \tilde{\mathbf{e}}(\mathbf{r})} \\
& =i \omega \mu_{0} g \tilde{\mathbf{j}}(\mathbf{r})
\end{aligned}
$$

The solution of (24) is well-known as [12]

$$
\tilde{\mathbf{e}}(\mathbf{r})=i \omega \mu_{0} g \iiint \underline{\underline{g}}(\mathbf{r}, \mathbf{s}) \cdot \tilde{\mathbf{j}}(\mathbf{s}) d^{3} \mathbf{s},
$$

where

$$
\underline{\underline{g}}(\mathbf{r}, \mathbf{s})=\left(\underline{\underline{I}}+\frac{\nabla \nabla}{k_{0}^{2} g^{2}}\right) \frac{\exp \left(i k_{0} g|\mathbf{r}-\mathbf{s}|\right)}{4 \pi|\mathbf{r}-\mathbf{s}|} .
$$

In order to go back from (25) to (9), we have to invert the two transformations in reverse sequence: Substitution of (18) and (20) in (25) yields

$$
\begin{aligned}
\mathbf{e}(\mathbf{r}) & =i \omega \mu_{0}\left(\operatorname{adj} \underline{\underline{\gamma}}^{1 / 2}\right) \\
& \cdot\left(\iiint \underline{\underline{g}}(\mathbf{r}, \mathbf{s}) \cdot \mathbf{j}(\mathbf{s}) d^{3} \mathbf{s}\right),
\end{aligned}
$$

wherein

$$
\begin{array}{r}
\underline{\underline{g}}(\mathbf{r}, \mathbf{s})=\left(\underline{\underline{I}}+\frac{1}{k_{0}^{2} g^{2}} \underline{\underline{\gamma}} \cdot \nabla \nabla\right) \\
\frac{\exp \left[i k_{0} g|\underline{\underline{\gamma}}-1 / 2 \cdot(\mathbf{r}-\mathbf{s})|\right]}{4 \pi\left|\underline{\gamma}^{-1 / 2} \cdot(\mathbf{r}-\mathbf{s})\right|} .
\end{array}
$$

By substituting for $\mathbf{e}$ and $\mathbf{j}$ in (27) using (12) and (14), respectively, we find

$$
\begin{gathered}
\mathbf{E}(\mathbf{r})=i \omega \mu_{0} \exp \left(i k_{0} \boldsymbol{\Gamma} \cdot \mathbf{r}\right)\left(\operatorname{adj} \underline{\underline{\gamma}} \underline{\gamma}^{1 / 2}\right) \\
\cdot\left(\iint \underline{\underline{g}}(\mathbf{r}, \mathbf{s}) \cdot \mathbf{J}(\mathbf{s})\right. \\
\left.\exp \left(-i k_{0} \boldsymbol{\Gamma} \cdot \mathbf{s}\right) d^{3} \mathbf{s}\right) .
\end{gathered}
$$

Therefore, the dyadic Green function $\underline{\underline{G}}_{e}(\mathbf{r}, \mathbf{s})$ emerges from (29) as

$$
\begin{gathered}
\underline{\underline{G}}_{e}(\mathbf{r}, \mathbf{s})=\exp \left[i k_{0} \mathbf{\Gamma} \cdot(\mathbf{r}-\mathbf{s})\right]\left(\operatorname{adj} \underline{\underline{\gamma}}^{1 / 2}\right) \\
\cdot\left(\underline{\underline{I}}+\frac{1}{k_{0}^{2} g^{2}} \underline{\underline{\gamma}} \cdot \nabla \nabla\right) \\
\frac{\exp \left[i k_{0} g\left|\underline{\underline{\gamma}}^{-1 / 2} \cdot(\mathbf{r}-\mathbf{s})\right|\right]}{4 \pi\left|\underline{\underline{\gamma}}^{-1 / 2} \cdot(\mathbf{r}-\mathbf{s})\right|} \cdot
\end{gathered}
$$

Equation (30) is the desired result. If $\underline{\underline{\gamma}}=\underline{\underline{I}}$ and $\boldsymbol{\Gamma}=\mathbf{0}$, this expression reduces to the usual dyadic Green function for gravitationally unaffected vacuum [12].

\section{References}

[1] Jackson J D 1999 Classical Electrodynamics, 3rd ed (New York: Wiley) ch 6

[2] Lakhtakia A and Messier R 2005 Sculptured Thin Films: Nanoengineered Morphology and Optics (Bellingham, WA, USA: SPIE Press) ch 6

[3] Kraus J D 1984 Electromagnetics, 3rd ed (New York: McGraw-Hill)

[4] Schutz B F 1985 A First Course in General Relativity (Cambridge, UK: Cambridge University Press)

[5] Plebanski J 1960 Phys. Rev. 1181396

[6] Mackay T G, Lakhtakia A and Setiawan S 2005 New J. Phys. 775

[7] Elliott R S 1993 Electromagnetics: History, Theory, and Applications (New York: IEEE Press)

[8] Gersten J I and Smith F W 2001 The Physics and Chemistry of Materials (New York: Wiley)

[9] Weiglhofer W S and Lakhtakia A (eds) 2003 Introduction to Complex Mediums for Optics and Electromagnetics (Bellingham, WA, USA: SPIE Press)

[10] Lakhtakia A and Weiglhofer W S 1997 Microw. Opt. Technol. Lett. 15168 
[11] Lakhtakia A and Weiglhofer W S 1994 Int. J. Appl. Electromag. Mater. 5101

[12] Chen H C 1983 Theory of Electromagnetic Waves (New York: McGraw-Hill) ch 9 